\title{
Teleportation of qubit states through dissipative channels: Conditions for surpassing the no-cloning limit
}

\author{
Şahin Kaya Özdemir* ${ }^{1,2,3}$ Karol Bartkiewicz ${ }^{*}{ }^{4}$ Yu-xi Liu, ${ }^{5,6}$ and Adam Miranowicz ${ }^{4,1,3}$ \\ ${ }^{1}$ SORST Research Team for Interacting Carrier Electronics, \\ 4-1-8 Honmachi, Kawaguchi, Saitama 331-0012, Japan \\ ${ }^{2}$ CREST Research Team for Photonic Quantum Information, \\ 4-1-8 Honmachi, Kawaguchi, Saitama 331-0012, Japan \\ ${ }^{3}$ Graduate School of Engineering Science, Osaka University, Toyonaka, Osaka 560-8531, Japan \\ ${ }^{4}$ Institute of Physics, Adam Mickiewicz University, 61-614 Poznań, Poland \\ ${ }^{5}$ Frontier Research System, Institute of Physical and Chemical Research (RIKEN), Wako-shi 351-0198, Japan \\ ${ }^{6}$ CREST, Japan Science and Technology Agency (JST), Kawaguchi, Saitama 332-0012, Japan
}

(Dated: October 27, 2018)

\begin{abstract}
We investigate quantum teleportation through dissipative channels and calculate teleportation fidelity as a function of damping rates. It is found that the average fidelity of teleportation and the range of states to be teleported depend on the type and rate of the damping in the channel. Using the fully entangled fraction, we derive two bounds on the damping rates of the channels: one is to beat the classical limit and the second is to guarantee the non-existence of any other copy with better fidelity. Effect of the initially distributed maximally entangled state on the process is presented; and the concurrence and the fully entangled fraction of the shared states are discussed. We intend to show that prior information on the dissipative channel and the range of qubit states to be teleported is helpful for the evaluation of the success of teleportation, where success is defined as surpassing the fidelity limit imposed by the fidelity of 1-to-2 optimal cloning machine for the specific range of qubits.
\end{abstract}

PACS numbers: 03.67.Hk, 03.65.Ud, 03.65.Ta

\section{INTRODUCTION}

The quantum state of a system can be transmitted from a location to a distant one using only classical information provided that a quantum channel exists between the sender and the receiver. Sharing entangled states between the two parties opens the necessary quantum channel [1]. Research in quantum state transfer [2], especially the quantum teleportation [3], has emerged as one of the major research areas of theoretical and experimental quantum mechanics. Various discussions and criteria have appeared about the evaluation of the state transfers under ideal and imperfect conditions [4]. In a perfect scheme, the shared entangled state is a maximally entangled state (MES) enabling perfect quantum state transfer. However, in practice, entanglement is susceptible to local interactions with the environment, which can result in loss of coherence. In this article, we study the teleportation of qubits through damping channels.

We consider quantum state transfer as an operation, such as cloning and teleportation, which beats the classical limits on measurement and transmission. The resemblance of two quantum states and the properties of quantum state transfer (teleportation and cloning) are quantified by the fidelity $F\left(\left|\psi_{\text {in }}\right\rangle\right)=\left\langle\psi_{\text {in }}\left|\hat{\rho}_{\text {out }}\right| \psi_{\text {in }}\right\rangle$, which measures the overlap of the states $\left|\psi_{\text {in }}\right\rangle$ to be teleported (cloned) and the output state with the density operator

*These authors have made equal contribution. $\hat{\rho}_{\text {out }}$.

A qubit state to be teleported $\left|\psi_{\text {in }}\right\rangle=\alpha|0\rangle+\beta|1\rangle$ with $|\alpha|^{2}+|\beta|^{2}=1$ can be represented on a Bloch sphere as

$$
\left|\psi_{\text {in }}\right\rangle=\cos (\delta / 2) \mathrm{e}^{i \gamma}|0\rangle+\sin (\delta / 2)|1\rangle,
$$

where $\delta$ and $\gamma$ are the polar and azimuthal angles, respectively. Since this state is generally unknown, it is more appropriate to calculate the average of the fidelity $F\left(\left|\psi_{\text {in }}\right\rangle\right)$ over all possible states $\left|\psi_{\text {in }}\right\rangle$ to quantify the process. This average fidelity $F=\overline{\left\langle\psi_{\text {in }}\left|\hat{\rho}_{\text {out }}\right| \psi_{\text {in }}\right\rangle}[5]$ can be calculated as

$$
F=\frac{1}{4 \pi} \int_{0}^{2 \pi} d \gamma \int_{0}^{\pi} d \delta F(\delta, \gamma) \sin \delta
$$

where the $4 \pi$ is the solid angle.

The relation between the teleportation fidelity and the degree of entanglement shared by the parties has been studied by many researchers (e.g. in [3, 4, 4, 5, 6, 7, 7, 8, 9, 10] and others cited in [1]) and it has been shown that (i) less entangled quantum channel reduces the fidelity and the range of states, which can be teleported [3], (ii) for the standard teleportation scheme, the maximum attainable average fidelity is simply related to the fully entangled fraction of a bipartite entangled state [6], and (iii) some mixed states, which do not violate the Bell inequalities, can still be used for teleportation 5]. On the other hand, only a few studies are directed to the relation between the fidelity of teleportation and the type and strength of the damping in the quantum channel. That is the topic of the present study. 
According to the definition of teleportation as stated by Bennett et al. [3], in the process of quantum teleportation, one can construct an exact replica of the original unknown quantum state with the cost of destroying the original state. Therefore, to call a quantum state transfer operation as quantum teleportation, the process should not only generate output states with better qualities than what can be done classically but also obey the no-cloning theorem [11]. Defining a teleportation operator $\hat{U}_{\text {tel }}$, which can be implemented in a standard quantum circuit (see, e.g., 12]) with an input state $\hat{\rho}_{\text {in }}=\hat{\rho}_{a}$ and a shared entangled state $\hat{\rho}_{\text {ent }}=\hat{\rho}_{b, c}$, the output state $\hat{\rho}_{\text {out }}$ is written as

$$
\hat{\rho}_{\text {out }}=\operatorname{Tr}_{\text {in }, \mathrm{a}}\left[\hat{U}_{\text {tel }} \hat{\rho}_{\text {in }} \otimes \hat{\rho}_{\text {ent }} \hat{U}_{\text {tel }}^{\dagger}\right] .
$$

If the teleportation process is ideal then $\hat{\rho}_{\text {out }}=\hat{\rho}_{\text {in }}$ implying a fidelity value of unity. However, in practical applications, this is not the case due to the presence of noise which may be due to (i) noisy sources of $\hat{\rho}_{\text {in }}$ and $\hat{\rho}_{\text {ent }}$, (ii) noisy entanglement distribution channel, (iii) noisy measurements and unitary operators, and (iv) an eavesdropper who attempts to clone $\hat{\rho}_{\text {in }}$. Since, in general one cannot be sure of which of the above are the reason, all the noise in the process should be attributed to an eavesdropper in order to assess the security whenever quantum teleportation is to be used as a means of secure communication. This assessment to quantify the process should be done according to the definition of the teleportation given above. That is, one should check to see whether $F$ in Eq. (2) satisfies the conditions of (i) beating the classical limit, and (ii) obeying the no-cloning.

The linearity of quantum mechanics forbids the exact cloning of an unknown quantum state, however, if one allows discrepancies between the original quantum state and its copy, then it is possible to devise a scheme that can produce clones and copies of a given unknown state with the highest resemblance to the original one [13, 14, 15, 16] (for reviews see [2]). This is known as the optimal cloning, where with the increasing number of clones (copies), the resemblance to the original state decreases. It has been shown that for a state-independent universal cloning machine the relation between the optimum fidelity $F$ of each copy and the number $M$ of copies is given by $F=(2 M+1) /(3 M)$. In classical situations, one can make infinite number of copies $(M \rightarrow \infty)$ of a given state resulting in a fidelity $F=2 / 3$, which is the best one can do with classical operations. On the other hand, when $M=2$, the universal cloning machine has an optimum fidelity of $F=5 / 6[13,14,15,16]$.

Combining the above information on teleportation and cloning, one can infer that a teleportation process beats the classical limit if $F>2 / 3$, and obeys the no-cloning requirement if $F>5 / 6[13,14,15,16]$. If this is assured, then there is no any other copy of the output state with better fidelity, therefore, the teleportation process is secure. It is noteworthy that this is true if and only if the quantum state $\hat{\rho}_{\text {in }}$ is completely unknown to the eavesdropper. In some cases, $\hat{\rho}_{\text {in }}$ may be prepared in a state that is selected from a known ensemble of states. If the eavesdropper has this a priori knowledge about $\hat{\rho}_{\text {in }}$, a state dependent cloner which can perform better than the optimal universal one can be constructed. Thus, the fidelity constraint imposed on teleportation due to nocloning condition will become much stricter.

Quality of the shared entangled state is a good criterion to quantify the reliability of the quantum teleportation. Bennett et al. [17] and, in general case, Horodecki et al. [6, 18] (for a review see [1]) have shown that for a shared bipartite entangled state $\hat{\rho}_{\text {ent }}$ to be useful for quantum teleportation, its fully entangled fraction $f_{\text {ent }}$, defined by [19]

$$
f_{\text {ent }}=\max _{\Phi}\left\langle\Phi\left|\hat{\rho}_{\text {ent }}\right| \Phi\right\rangle,
$$

must be greater than $1 / 2$. In Eq. (44), maximum is taken over all MES $|\Phi\rangle$. It has also been shown that, the maximum achievable teleportation fidelity $F$ is related to $f_{\text {ent }}$ by [6]

$$
F=\frac{2 f_{\mathrm{ent}}+1}{3}
$$

States with $f_{\text {ent }} \leq 1 / 2$ cannot be used directly for teleportation unless they are enhanced through filtering to satisfy $f_{\text {ent }}>1 / 2$. Choosing the boundary value of $f_{\text {ent }}=1 / 2$ gives a teleportation fidelity of $F=2 / 3$ which is the boundary between classical and quantum state transfer. That is if $f_{\text {ent }} \leq 1 / 2$ and hence $F \leq 2 / 3$, then the same operation can be done classically. According to this definition, if in a process $F>2 / 3$ is achieved then it can be called quantum teleportation. On the other hand, the discussion on cloning in the former paragraphs implies that one can make infinite number of copies of a qubit with a fidelity of $2 / 3$ which violates the original definition of quantum teleportation given by Bennett et al. [3]. Here again arises the question of achievable teleportation fidelity, which guarantees better than classical teleportation and surpasses the no-cloning limit.

The problem studied in this paper can be formulated as follows: Alice and Bob are far from each other, and they share an entangled quantum state $\hat{\rho}_{\text {en }}$. The entangled state is prepared either by a third party, say Claire, and delivered to Alice and Bob (scenario 1: two-qubit affected scenario) or prepared by Alice and one of the qubits is sent to Bob and the other is kept with her (scenario 2: one-qubit affected scenario) as shown in Fig. 11 The only manipulations that Alice and Bob are allowed to do is local quantum operations and classical communications. Now suppose that Alice wants to transfer the quantum state represented by the qubit state $\left|\psi_{\text {in }}\right\rangle$ to Bob and the entangled state is distributed through a dissipating channel. Then, how does the dissipation of the channel affect the entanglement properties of the distributed entangled state, and hence what is its effect on the transferred quantum state? What is the allowable amount of dissipation that does not affect the security of quantum state transfer? 

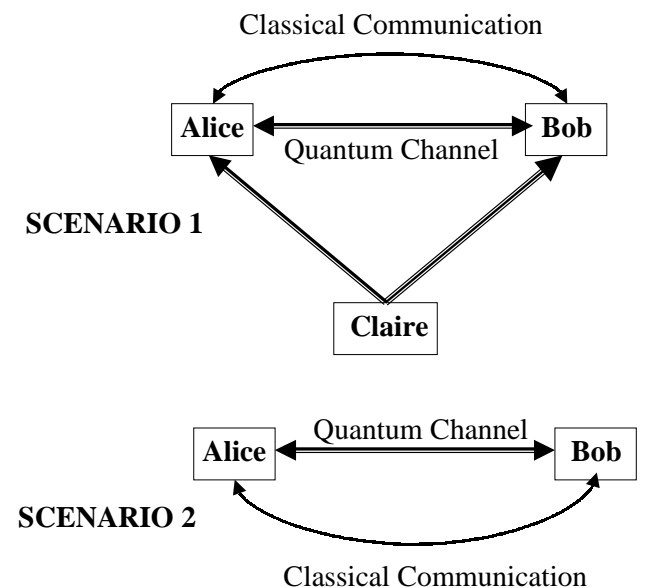

FIG. 1: Teleportation scenario 1, where both qubits of $\hat{\rho}_{\text {ent }}$ are affected by the channel, and scenario 2, where only one of the qubits is affected. Quantum channel is formed by the shared entangled state.

In this paper, we derive the damping rates of quantum channels at which a quantum state transfer that overcomes the classical counterpart can be realized. In the same way, conditions, which guarantee a secure quantum teleportation, are also derived. We study the effect of noise on the range of qubits that can be teleported accurately. The noisy channels, including amplitude damping channel, phase damping channel and depolarizing channel, and the effects of these noisy channels on the distributed entanglement and teleportation process are studied in Sec. II and III. And finally, Sec. IV includes a brief summary and conclusion of this study.

\section{EFFECT OF DAMPING CHANNELS ON ENTANGLEMENT AND TELEPORTATION}

We consider the two scenarios shown in Fig. 11 In the first scenario, the qubits of the initial MES are distributed through two channels, which may or may not have the same damping properties. On the other hand, in the second scenario, only one of the qubits of the initial MES is distributed through the damping channel. In the following, we give analytical expressions, which show how a given state is affected when transmitted through noisy channels causing amplitude damping, phase damping or depolarization. Initial MES that are considered in this study are the Bell states

$$
\begin{aligned}
\left|\psi^{ \pm}\right\rangle & =\frac{1}{\sqrt{2}}(|01\rangle \pm|10\rangle), \\
\left|\phi^{ \pm}\right\rangle & =\frac{1}{\sqrt{2}}(|00\rangle \pm|11\rangle) .
\end{aligned}
$$

We derive the bounds for the damping rate of the channel to satisfy the quantum teleportation conditions discussed in the previous section. In the following, we assume that there is no a priori information on $\hat{\rho}_{\text {in }}$, therefore the optimal universal cloning machine which imposes $F>5 / 6$ is considered.

\section{A. Amplitude Damping Channel}

The evolution of environment (denoted by subscript $e$ ) and a system (subscript $a$ or, equivalently, $b$ ) with the states $|0\rangle$ and $|1\rangle$ is defined by the following transformation in the presence of the amplitude damping channel (ADC) 20]:

$$
\begin{aligned}
|0\rangle_{a}|0\rangle_{e} & \rightarrow|0\rangle_{a}|0\rangle_{e} \\
|1\rangle_{a}|0\rangle_{e} & \rightarrow \sqrt{q}|1\rangle_{a}|0\rangle_{e}+\sqrt{p}|0\rangle_{a}|1\rangle_{e}
\end{aligned}
$$

where $q \equiv 1-p$. This transformation implies that a system with an excited state makes a transition to the ground state with a probability $p$ and emits a photon to the environment which makes a transition to the excited state. When the system is initially in the ground state, there is no transition.

\section{Input Bell states $\left|\psi^{ \pm}\right\rangle$}

If both of the qubits in the Bell states $\left|\psi^{ \pm}\right\rangle$are transmitted through an ADC (scenario 1), then using Eq. (7), we can write the state at the output of the channel as

$$
\begin{aligned}
\left|\Psi^{ \pm}\right\rangle_{a b e_{1} e_{2}=}= & \frac{1}{\sqrt{2}}\left[\left(\sqrt{q_{b}}|01\rangle_{a b} \pm \sqrt{q_{a}}|10\rangle_{a b}\right)|00\rangle_{e_{1} e_{2}}\right. \\
& \left.+\left(\sqrt{p_{b}}|01\rangle_{e_{1} e_{2}} \pm \sqrt{p_{a}}|10\rangle_{e_{1} e_{2}}\right)|00\rangle_{a b}\right]
\end{aligned}
$$

where we assumed that channels have different damping rates denoted by $p_{a}, p_{b}$ and, for simplicity, we denote $q_{a} \equiv 1-p_{a}, q_{b} \equiv 1-p_{b}$. If we assume $p_{a}=p_{b}=p$ and the environment is not monitored (unwatched channel), the shared state between Alice and Bob at the outputs of the channels can be found by tracing out the environment variables resulting in $\hat{\rho}_{a b}^{ \pm}=q\left|\psi^{ \pm}\right\rangle_{a b a b}\left\langle\psi^{ \pm}\right|+$ $p|00\rangle_{a b a b}\langle 00|$. It is seen that the MES survives with a probability of $q$. On the other hand, if the environment is monitored, Alice and Bob proceed with the protocol if no photon is detected in the environment implying they have a MES, and they do nothing when photon is detected in the environment.

If only one of the qubits (say, that for Bob) is sent through the channel (scenario 2), the damping in the channel affects only that part. If the channel is watched and no photon is detected in the environment, the state that is shared between Alice and Bob becomes

$$
\left|\Psi^{ \pm}\right\rangle_{a b}=\frac{1}{\sqrt{2-p_{b}}}\left( \pm \sqrt{q_{b}}|01\rangle_{a b}+|10\rangle_{a b}\right) .
$$


For an unwatched channel, the shared state is given as

$$
\hat{\rho}_{a b}^{ \pm}=\frac{1}{2}\left[\left(2-p_{b}\right)\left|\Psi^{ \pm}\right\rangle_{a b a b}\left\langle\Psi^{ \pm}\left|+p_{b}\right| 00\right\rangle_{a b a b}\langle 00|\right] .
$$

It is clearly seen that if only one qubit of the initial MES is sent through the ADC, the shared state between the parties is no longer a MES.

Using Eq. (8), one can find that for scenario 1, the fully entangled fraction is [8]

$$
f_{\mathrm{ent}, 1}^{ \pm}=\frac{1}{4}\left(\sqrt{q_{a}}+\sqrt{q_{b}}\right)^{2}
$$

if $p_{a} \geq \frac{1}{2}\left(q_{b}+\sqrt{1+2 p_{b}-3 p_{b}^{2}}\right)$ is satisfied, otherwise it becomes

$$
f_{\mathrm{ent}, 2}^{ \pm}=\frac{1}{4}\left(p_{a}+p_{b}\right)
$$

If we assume that both channels have the same damping properties that is $p_{a}=p_{b}=p$, the fully entangled fraction is found as

$$
f_{\mathrm{ent}}^{ \pm}=\left\{\begin{array}{cc}
q & \text { if } p \leq 2 / 3 \\
p / 2 & \text { if } p>2 / 3 .
\end{array}\right.
$$

For scenario 2, where $p_{a}=0, f_{\mathrm{ent}}^{ \pm}$becomes $f_{\mathrm{ent}}^{ \pm}=\frac{1}{4}(1+$ $\left.\sqrt{q_{b}}\right)^{2}$ for all $p_{b}$.

Imposing the condition $f_{\text {ent }}>1 / 2$, which assures that a quantum state operation beats the classical limit, gives the relation $\sqrt{q_{a}}+\sqrt{q_{b}}>\sqrt{2}$ for scenario 1 [8]. Taking $p_{b}$ as a variable, it can be found that $p_{a}<2(\sqrt{2}-1)$ and $p_{b} \leq p_{a}-2+2 \sqrt{2 q_{a}}$ must be satisfied simultaneously [8]. Similarly for the case $p_{a}=p_{b}=p$, one can find that classical limit can be beaten only when $p<1 / 2$. For scenario 2 , it can easily be shown that $p_{b}<2(\sqrt{2}-$ 1) must be satisfied. If the channels are watched but no photon is detected, then $f_{\text {ent }}>1 / 2$ can always be satisfied provided that $p_{a}<1 \wedge p_{b}<1$ and $p_{b}<1$ for scenarios 1 and 2 , respectively.

If the conditions given in the above paragraph for unwatched channels are satisfied, one can only be sure that the operation is a quantum one with fidelity $F>2 / 3$, however, cannot be sure about the security of the process, which requires $F>5 / 6$ according to $1 \rightarrow 2$ cloning condition. Then solving Eq. (5) for $f_{\text {ent }}$ to satisfy $F>5 / 6$, we find that

$$
f_{\text {ent }}>\frac{3}{4}
$$

must be satisfied for the fully entangled fraction. Imposing this condition on the shared entangled state between the two parties results in a much tighter condition on the channel damping rates, which can be summarized as follows

$$
f_{\text {ent }}^{ \pm}>\frac{3}{4} \text { if }
$$

$$
\left\{\begin{array}{cc}
p<\frac{1}{4} & \text { scenario } 1 \\
& \text { for } p_{b}=p_{a}=p \\
p_{b}<p_{a}-3+2 \sqrt{3 q_{a}} & \text { scenario } 1 \\
p_{a} \leq 2 \sqrt{3}-3 \text { or vice versa } & \text { for } p_{b} \neq p_{a}, \\
p_{b}<2 \sqrt{3}-3 & \text { scenario } 2 .
\end{array}\right.
$$

\section{Input Bell states $\left|\phi^{ \pm}\right\rangle$}

In the scenario 1, when both qubits of $\left|\phi^{ \pm}\right\rangle$are sent through the damping channels, the shared state between Alice and Bob for watched and unwatched channel are found as

$$
\left|\Phi^{ \pm}\right\rangle_{a b}=\frac{|00\rangle_{a b} \pm \sqrt{q_{a} q_{b}}|11\rangle_{a b}}{\sqrt{1+q_{a} q_{b}}}
$$

and

$$
\begin{aligned}
\hat{\rho}_{a b}^{ \pm}= & \frac{1}{2}\left[\left(1+q_{a} q_{b}\right)\left|\Phi^{ \pm}\right\rangle_{a b a b}\left\langle\Phi^{ \pm}\right|\right. \\
& +q_{b} p_{a}|01\rangle_{a b a b}\left\langle 01\left|+p_{b} q_{a}\right| 10\right\rangle_{a b a b}\langle 10| \\
& \left.+p_{a} p_{b}|00\rangle_{a b a b}\langle 00|\right],
\end{aligned}
$$

respectively, where we have considered that no photon is detected in the environment for the watched channel case. From these equations, it is seen that a MES survives with a non-zero probability iff $p_{a}=p_{b}=0$.

When only one of the qubits (say again, Bob's qubit) of the MES is propagated through the ADC, the shared state between Alice and Bob is not maximally entangled unless $p_{b}=0$ for both watched and unwatched channels as can be seen in the following expressions given, respectively, for watched and unwatched channels

$$
\left|\Phi^{ \pm}\right\rangle_{a b}=\frac{1}{\sqrt{2-p_{b}}}\left(|00\rangle_{a b} \pm \sqrt{q_{b}}|11\rangle_{a b}\right),
$$

and

$$
\hat{\rho}_{a b}^{ \pm}=\frac{1}{2}\left[\left(2-p_{b}\right)\left|\Phi^{ \pm}\right\rangle_{a b a b}\left\langle\Phi^{ \pm}\left|+p_{b}\right| 10\right\rangle_{a b a b}\langle 10|\right] .
$$

Then the fully entangled fraction of the shared state, when the channel is not watched, is found as

$$
f_{\mathrm{ent}}^{ \pm}=\frac{1}{4}\left[p_{a} p_{b}+\left(1+\sqrt{q_{a} q_{b}}\right)^{2}\right]
$$

which reduces to

$$
f_{\text {ent }}^{ \pm}=\frac{1}{4}\left\{\begin{array}{cc}
2\left(p^{2}-2 p+2\right) & \text { scenario } 1 \\
& \text { for } p_{a}=p_{b} \equiv p \\
\left(1+\sqrt{q_{b}}\right)^{2} & \text { scenario } 2
\end{array}\right.
$$

It can easily be found from Eq. (21) that, the condition $f_{\text {ent }}>1 / 2$ is satisfied for any $p$ in the range $p<1$ when both channels have the same damping rates in scenario 1 ; 
and for $p_{b}<2(\sqrt{2}-1)$ in scenario 2 . When the channels have different damping rates, we can write using Eq. (20) that $p_{a} p_{b}+\left(1+\sqrt{q_{a} q_{b}}\right)^{2}>2$ must be satisfied to beat the classical limit. Analytical solution for this is very lengthy to give here. Instead, to give an idea on the relation between $p_{b}$ and $p_{a}$ to satisfy the condition $f_{\text {ent }}>1 / 2$, we give some numerical values: when $p_{b}=1 / 2, p_{a}<$ $7 / 8$ and when $p_{b}=1 / 4, p_{a}$ must satisfy $p_{a}<(6 \sqrt{6}-$ $13) / 2$ to beat the classical limit. As it has been pointed out by Bandyopadhyay [8], scenario 1 can be made to have higher $f_{\text {ent }}$ than scenario 2 such that $f_{\text {ent }}>1 / 2$ is satisfied. This, in turn, implies that for the state $\left|\phi^{ \pm}\right\rangle$, one can let one of the qubits to undergo a controlled dissipation if the information on the dissipation of the other qubit in the other channel is available.

Looking at the condition $f_{\text {ent }}>3 / 4$ for quantum teleportation to surpass the no-cloning limit, we find the following constraints on the damping rates of the ADC:

$$
\begin{aligned}
& f_{\mathrm{ent}}^{ \pm}>\frac{3}{4} \text { if } \\
& \left\{\begin{array}{cc}
p<1-\frac{\sqrt{2}}{2} & \text { scenario } 1 \\
& \text { for } p_{a}=p_{b} \equiv p, \\
p_{a} \leq 2 \sqrt{3}-3 & \text { scenario } 1 \\
p_{b} \leq g\left(p_{a}\right) \text { or vice versa } & \text { for } p_{a} \neq p_{b}, \\
p_{a}<2 \sqrt{3}-3 & \text { scenario } 2,
\end{array}\right.
\end{aligned}
$$

where $g(x)=(1-2 x)^{-2}[-3+x(3+2 x)+$ $\left.2 \sqrt{(1-x)\left(2 x^{2}-6 x+3\right)}\right]$. Contrary to the above case, a controlled dissipation cannot increase $f_{\text {ent }}$ above $3 / 4$.

\section{B. Phase Damping Channel}

A phase damping channel (PDC) affects an input state with the following transformations [20]

$$
\begin{aligned}
|0\rangle_{a}|0\rangle_{e} & \rightarrow \sqrt{q}|0\rangle_{a}|0\rangle_{e}+\sqrt{p}|0\rangle_{a}|1\rangle_{e}, \\
|1\rangle_{a}|0\rangle_{e} & \rightarrow \sqrt{q}|1\rangle_{a}|0\rangle_{e}+\sqrt{p}|1\rangle_{a}|2\rangle_{e} .
\end{aligned}
$$

In this channel, the energy of the information carrier is conserved (no losses to environment), however the state of the carrier is decohered.

\section{Input Bell states $\left|\psi^{ \pm}\right\rangle$}

Bell states $\left|\psi^{ \pm}\right\rangle$evolve into

$$
\begin{gathered}
\hat{\rho}_{a b}^{ \pm}=\frac{1}{2}\left(1-q_{a} q_{b}\right)\left(|01\rangle_{a b a b}\langle 01|+| 10\rangle_{a b a b}\langle 10|\right) \\
+q_{a} q_{b}\left|\psi^{ \pm}\right\rangle_{a b a b}\left\langle\psi^{ \pm}\right|
\end{gathered}
$$

when both qubits are sent through the unwatched channel. For the limiting case, $p_{a}=1 \vee p_{b}=1$, off-diagonal components of the density matrix vanish resulting in a mixed state. For a watched channel with no photon detected in the environment, there is a probability of $q_{a} q_{b}$ that the state observed is $\left|\psi^{ \pm}\right\rangle$.

On the other hand, when only one qubit is sent (scenario 2), the probability that the MES survives becomes $q_{b}$ when the channel is watched. When the channel is not watched, then the output state, which is mixed and not a MES, can be found from Eq. (24) by substituting $p_{a}=0$.

When the $f_{\text {ent }}^{ \pm}$of the output state at the end of the unwatched channels are calculated it is seen that

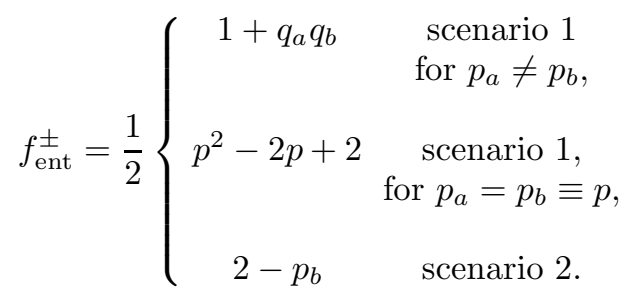

Then we find that $f_{\text {ent }}^{ \pm}$is always greater than $1 / 2$ provided that $p_{b} \neq 1 \wedge p_{a} \neq 1$ and $p \neq 1$ are satisfied for both scenarios. Moreover, we find scenario 1 cannot be made to have $f_{\text {ent }}^{ \pm}$larger than scenario 2 .

The no-cloning limit imposes the following conditions on the allowable $\mathrm{PDC}$ rate:

$$
\begin{aligned}
& f_{\text {ent }}^{ \pm}>\frac{3}{4} \text { if } \\
& \left\{\begin{array}{cc}
p<1-\frac{\sqrt{2}}{2} & \begin{array}{c}
\text { scenario } 1 \\
\text { for } p_{a}=p_{b} \equiv p
\end{array} \\
p_{b}<\left(1-2 p_{a}\right) /\left(2 q_{a}\right) & \text { scenario } 1 \\
p_{a}<1 / 2 \text { or vice versa } & \text { for } p_{a} \neq p_{b}, \\
p_{b}<1 / 2 & \text { scenario } 2 .
\end{array}\right.
\end{aligned}
$$

$$
\text { 2. Input Bell states }\left|\phi^{ \pm}\right\rangle
$$

When the input is $\left|\phi^{ \pm}\right\rangle$, then the state at the output of the channels becomes

$$
\begin{gathered}
\hat{\rho}_{a b}^{ \pm}=\frac{1}{2}\left(1-q_{a} q_{b}\right)\left(|00\rangle_{a b a b}\langle 00|+| 11\rangle_{a b a b}\langle 11|\right) \\
+q_{a} q_{b}\left|\phi^{ \pm}\right\rangle_{a b a b}\left\langle\phi^{ \pm}\right|
\end{gathered}
$$

for an unwatched channel for scenario 1. A comparison of this output state with Eq. (24) reveals that the same discussions and the conditions on the channel damping properties are valid here, too.

\section{Depolarizing Channel}

When a qubit is sent through a depolarizing channel (DC), with a probability $q \equiv 1-p$ it is intact, while with 
probability $p$ an error (bit flip error, phase flip error or both) occurs. The transformation that characterizes this channel is [20]

$$
\begin{aligned}
|0\rangle_{a}|0\rangle_{e} & \rightarrow \sqrt{1-\frac{3 p}{4}}|0\rangle_{a}|0\rangle_{e} \\
& +\sqrt{\frac{p}{4}}\left(|1\rangle_{a}|1\rangle_{e}+i|1\rangle_{a}|2\rangle_{e}+|0\rangle_{a}|3\rangle_{e}\right), \\
|1\rangle_{a}|0\rangle_{e} & \rightarrow \sqrt{1-\frac{3 p}{4}}|1\rangle_{a}|0\rangle_{e} \\
& +\sqrt{\frac{p}{4}}\left(|0\rangle_{a}|1\rangle_{e}-i|0\rangle_{a}|2\rangle_{e}-|1\rangle_{a}|3\rangle_{e}\right) .
\end{aligned}
$$

In the DC, any given state $|\varphi\rangle$ evolves to an ensemble of the four states $|\varphi\rangle, \hat{\sigma}_{x}|\varphi\rangle, \hat{\sigma}_{y}|\varphi\rangle$ and $\hat{\sigma}_{z}|\varphi\rangle$ where $\sigma_{k}$ is the Pauli operator. $p=1$ corresponds to complete depolarization where each of the four states occur with equal probabilities.

If the input state to the channel is $\left|\psi^{ \pm}\right\rangle$or $\left|\phi^{ \pm}\right\rangle$both qubits are sent through the channel then with a probability of $\left(4-3 p_{a}\right)\left(4-3 p_{b}\right) / 16$, this state is conserved at the output of the channels if the channel is watched and no photon is detected. For an unwatched channel with an input $\left|\eta_{1,2}^{ \pm}\right\rangle=\left|\psi^{ \pm}\right\rangle,\left|\phi^{ \pm}\right\rangle$for indices 1 and 2, respectively, the output state can be written as

$$
\begin{aligned}
\hat{\rho}_{a b}^{ \pm}= & \frac{1-q_{a} q_{b}}{4}\left(\left|\eta_{1,2}^{\mp}\right\rangle\left\langle\eta_{1,2}^{\mp}|+| \eta_{2,1}^{-}\right\rangle\left\langle\eta_{2,1}^{-}|+| \eta_{2,1}^{+}\right\rangle\left\langle\eta_{2,1}^{+}\right|\right) \\
& +\frac{1+3 q_{a} q_{b}}{4}\left|\eta_{1,2}^{ \pm}\right\rangle\left\langle\eta_{1,2}^{ \pm}\right|,
\end{aligned}
$$

which becomes a mixture of Bell states with equal probability $1 / 4$ when $p=1$. The effect of this channel on the input state when only one of the qubits is sent through can be found simply by substituting $p_{a}=0$. Imposing the criteria $f_{\text {ent }}>1 / 2$ and $f_{\text {ent }}>3 / 4$ on the state at the output of the channel for both scenarios, we find the following ranges for damping rate of the $\mathrm{DC}$

$$
\begin{aligned}
& f_{\text {ent }}^{ \pm}>\frac{1}{2} \text { if } \\
& \left\{\begin{array}{cc}
p<1-\sqrt{3} / 3 & \begin{array}{c}
\text { scenario } 1 \\
\text { for } p_{b}=p_{a}=p,
\end{array} \\
p_{a}<\frac{2-3 p_{b}}{3 q_{b}} ; p_{b}<2 / 3 & \text { scenario } 1 \\
& \text { for } p_{b} \neq p_{a}, \\
p_{b}<2 / 3 & \text { scenario } 2
\end{array}\right.
\end{aligned}
$$

and

$$
f_{\text {ent }}^{ \pm}>\frac{3}{4} \text { if }
$$

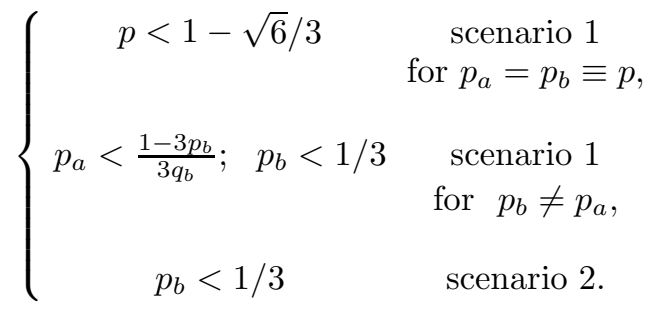

\section{Concurrence and fully entangled fraction}

Fully entangled fraction $f_{\text {ent }}$, given by (44), can be regarded as a measure of entanglement when the quantum channel is in a pure state and it is related to the Wootters concurrence $C$ [21] through the relation $f_{\text {ent }}=(1+C) / 2$. However, when the quantum channel is in a mixed state, $f_{\text {ent }}$ can no longer be used as a measure of entanglement. This is due to the fact that entanglement cannot be increased by local quantum operations and classical communications, but the fully entangled fraction $f_{\text {ent }}$ can be increased as shown by Bandyopadhyay [8] and Badziasg et al. [18].

In the following, we present the dependence of concurrence on the properties of the unwatched damping channels in both scenarios introduced previously and discuss the relation between $f_{\text {ent }}$ and concurrence so that we can assure a quantum state transfer and a secure quantum teleportation. As defined by Wootters [21], concurrence of a mixed state $\varrho$ is given by $C=$ $\max \left(0, \lambda_{1}-\lambda_{2}-\lambda_{3}-\lambda_{4}\right)$, where $\left\{\lambda_{i}\right\}$ are the squareroots of the eigenvalues, in decreasing order, of the nonHermitian matrix $\varrho \tilde{\varrho}$ with $\tilde{\varrho}=\hat{\sigma}_{y} \otimes \hat{\sigma}_{y} \varrho^{*} \hat{\sigma}_{y} \otimes \hat{\sigma}_{y}$, where Pauli matrices act on Alice and Bob qubit respectively and $(*)$ stands for complex conjugation.

For the ADC, we observe that, in the first scenario, the relations between the concurrence and the damping parameter are different for the initial entangled states $\left|\psi^{ \pm}\right\rangle$and $\left|\phi^{ \pm}\right\rangle$. The concurrence of the shared state at the output of the channels for the input state $\left|\psi^{ \pm}\right\rangle$is given as $C^{\prime}=\sqrt{q_{a} q_{b}}$. And the concurrence for $\left|\phi^{ \pm}\right\rangle$becomes $C^{\prime \prime}=\left(1-\sqrt{p_{a} p_{b}}\right) C^{\prime}$. When both channels have the same damping rate $p_{a}=p_{b} \equiv p$. It is seen that while $C^{\prime}$ decreases linearly with $p, C^{\prime \prime}$ decreases with $p^{2}$. On the other hand, for scenario 2 , both initial states show the same tendency, which is given as $C^{\prime}=C^{\prime \prime}=\sqrt{q}$.

In the cases of the PDC and DC, $C \equiv C^{\prime}=C^{\prime \prime}$. For the PDC, concurrence is found as $C=q_{a} q_{b}$ for the first scenario. The expression for the second scenario can be found by taking $p_{a}=0$ and $p_{b}=p$. Although the expressions found for concurrence for the ADC and PDC are valid for all values of $p_{a}$ and $p_{b}$ in the range of $[0,1]$, the expressions for concurrence in the case of the DC are valid only for a limited range of damping rates. For example, for the second scenario, concurrence is found as $C=1-3 p / 2$ provided that $p<2 / 3$, otherwise it is zero implying a separable state. For the first scenario when both DCs have the same damping rate, concurrence is given as $C=1+3 p(p-2) / 2$ when $p \leq 1-\sqrt{3} / 3$, oth- 
(i)

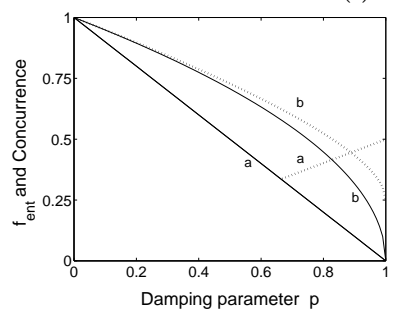

(iii)
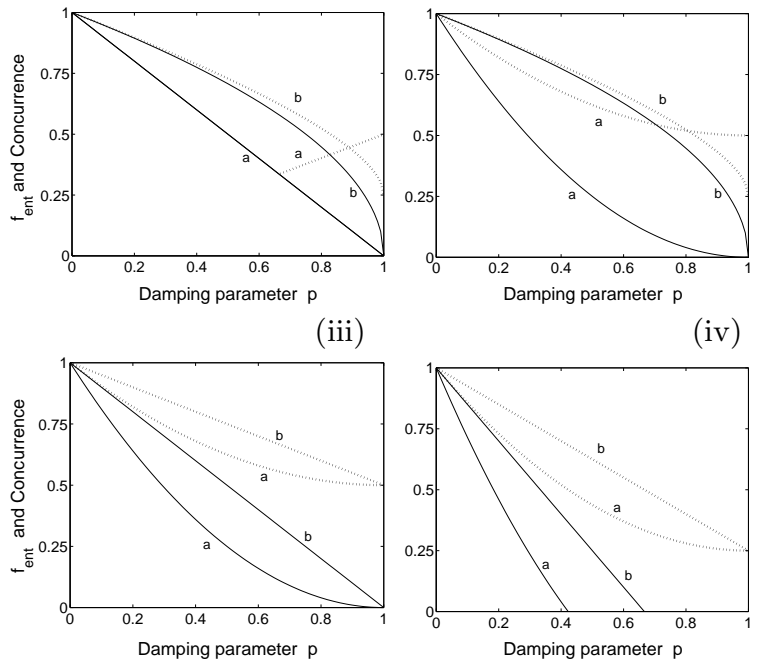

(iv)

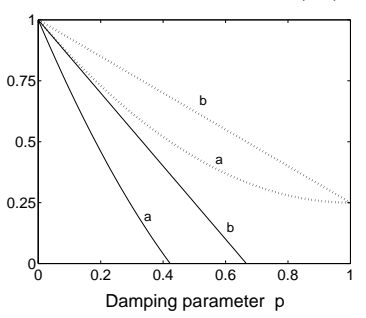

FIG. 2: Comparison of concurrence (solid curves) and fully entangled fraction (dashed curves) for the ADC, when the initial entangled state is $\left|\psi^{ \pm}\right\rangle$(i) and $\left|\phi^{ \pm}\right\rangle$(ii), as well as the PDC (iii) and the DC (iv) for scenarios 1 (curves a) and 2 (curves b). Note that for the PDC and DC results are independent of initial entangled state.

erwise $C=0$. When the damping rates are different, we find that $C=\left(3 q_{a} q_{b}-1\right) / 2$ provided that $p_{b}<2 / 3$ and $p_{a}<1-1 /\left(3 q_{b}\right)$ are satisfied simultaneously, otherwise $C=0$. It is seen from Fig. 2 that $f_{\text {ent }}$ is always $\leq 1 / 2$ for $C=0$. And even a very small amount of entanglement shifts the process from classical to quantum regime.

\section{RANGE OF QUBITS FOR ACCURATE TELEPORTATION}

In this section, we analyze the effect of the noise in the system on the range of qubits that can be teleported with a desired fidelity value. In order to show how the a priori information on the ensemble from which $\hat{\rho}_{\text {in }}$ is prepared affects the fidelity criterion on secure teleportation, we will consider an optimal one-to-two phase-covariant cloning machine (PCCM) 22, 23] in comparison with the universal cloning machine. We will assume that the states to be teleported are chosen from the whole set of qubit states with a fixed and specified polar angle $\delta$ in the Bloch sphere. An eavesdropper, who knows $\delta$, can use the optimal (one-to-two) PCCM for which the cloning fidelity is given by [23, 24]:

$$
\begin{aligned}
F^{\prime}(\delta) & =\frac{1}{2} \sin ^{2} \frac{\delta+\kappa \pi}{2}+\cos ^{4} \frac{\delta-\kappa \pi}{2}+\frac{\sqrt{2}}{4} \sin ^{2} \delta \\
& =\frac{1}{8}[5+\sqrt{2}+2 \cos (\delta+\kappa)-(\sqrt{2}-1) \cos (2 \delta)],
\end{aligned}
$$

where $\kappa=[[2 \delta / \pi]]$, i.e., $\kappa=0$ for $0 \leq \delta<\frac{\pi}{2}$ and $\kappa=1$ for $\frac{\pi}{2} \leq \delta \leq \pi$. Note that fidelity $F^{\prime}(\delta)$ for any $\delta$ is greater than the fidelity of the optimal universal cloning machine [13], given by $F=5 / 6$. For the qubit states on the equator of the Bloch sphere $(\delta=\pi / 2)$, the optimal PCCM prepares clones with fidelity $F^{\prime}(\pi / 2)=\frac{1}{4}(2+\sqrt{2})$. On the other hand, when the states are close to the poles that is in the neighborhood of $|1\rangle$ or $|0\rangle$ in the Bloch sphere, i.e., for a fixed angle $\delta=\pi-\Delta \delta$ or $\delta=0+\Delta \delta$ with $\Delta \delta \ll 1$, Eq. (33) simplifies to

$$
\begin{aligned}
F^{\prime}(\delta) & =\frac{1}{8}[5+\sqrt{2}+2 \cos \Delta \delta-(\sqrt{2}-1) \cos (2 \Delta \delta)] \\
& =1-\frac{3-2 \sqrt{2}}{8}(\Delta \delta)^{2}+\mathcal{O}(\Delta \delta)^{4} .
\end{aligned}
$$

In a teleportation process, measurement of Alice results in four possible outcomes $m_{i}$ where $i=0,1,2,3$ with $m_{0}=|00\rangle\left\langle 00\left|, m_{1}=\right| 01\right\rangle\left\langle 01\left|, m_{2}=\right| 10\right\rangle\langle 10|$, and $m_{3}=|11\rangle\langle 11|$. Then the state at Bob's side conditioned on Alice's measurement can be written as $\hat{\rho}^{\prime}\left(m_{i}\right)$. In the standard teleportation protocol with shared MES, upon receiving the classical information $i$, Bob can make the appropriate unitary operations on his qubit $\hat{\rho}^{\prime}\left(m_{i}\right)$ to obtain the teleported state $\hat{\rho}_{\text {out }}=\hat{\rho}_{\text {in }}$. We discuss how the measurement result affects this process in the presence of noise.

Since the entanglement distribution channel is noisy, the state at the output of teleportation process given in Eq. (3) can be rewritten as $\hat{\rho}_{\text {out }}=\operatorname{Tr}_{\text {in }, a}\left[\hat{U}_{\text {tel }} \hat{\rho}_{\text {in }} \otimes\right.$ $\left.\hat{\rho}_{\text {ent }}^{s} \hat{U}_{\text {tel }}^{\dagger}\right]$ where $\hat{\rho}_{\text {ent }}^{s}$ is the noisy entangled state. We can say that the fidelity is a function of $\delta, \gamma$ and the noise introduced into the system, and we can represent it as $F(\delta, \gamma) \equiv F\left(\left|\psi_{\text {in }}\right\rangle\right)$. We observe that $F(\delta, \gamma)$ is independent of $\gamma$, as denoted by $F(\delta) \equiv F(\delta, \gamma)$.

\section{A. Amplitude damping channel}

\section{Input Bell states $\left|\psi^{ \pm}\right\rangle$}

For the ADC, in scenario 1 , let us assume that $p_{b}=$ $p_{a}=p$ and Alice made a measurement, obtained the outcome $m_{1}$ and then sent the classical information $k=1$ to Bob. The output density operator conditioned on $m_{1}$ becomes $\hat{\rho}^{\prime}\left(m_{1}\right)=N\left[q \hat{\rho}_{\text {in }}+2 p \sin ^{2}(\delta / 2)|0\rangle\langle 0|\right]$ with $N$ being the renormalization constant defined as $N^{-1}=1-p \cos \delta$ and $\hat{\rho}_{\text {in }}$ is the density operator of the state to be teleported. Bob cannot rotate this $\hat{\rho}^{\prime}\left(m_{1}\right)$ to the desired state without the prior knowledge of $\delta$ and $\gamma$. Since $\left|\psi_{\text {in }}\right\rangle$ is supposed to be unknown, standard teleportation protocol fails to reproduce the desired state at Bob's side. This conclusion is valid for all $m_{i}$. Interestingly, output state at Bob's side can be grouped into two as $\chi_{0}=\left\{\hat{\rho}^{\prime}\left(m_{0}\right), \hat{\rho}^{\prime}\left(m_{2}\right)\right\}$ and $\chi_{1}=\left\{\hat{\rho}^{\prime}\left(m_{1}\right), \hat{\rho}^{\prime}\left(m_{3}\right)\right\}$. Although the output states in one of these groups can be rotated into each other by using a Z-gate or first X then Z-gate, states belonging to different groups cannot be rotated to each other. This problem is caused by the ADC, which reduces the degree of entanglement and introduces the additional terms $2 p \cos ^{2}(\delta / 2)|0\rangle\langle 0|$ and 
$2 p \sin ^{2}(\delta / 2)|0\rangle\langle 0|$, respectively, for $\chi_{0}$ and $\chi_{1}$. We observed that for teleportation in the presence of this ADC, if Alice's measurement yields $m_{1}$, Bob does not need to do anything. For other measurement results $m_{0}, m_{2}$ and $m_{3}$, Bob should apply $\hat{\sigma}_{x}, \hat{\sigma}_{y}$ and $\hat{\sigma}_{z}$, respectively. In this way, he rotates his qubit into the output state $\hat{\rho}_{\text {out }}\left(m_{k}\right)=N_{k}\left[q \hat{\rho}_{\text {in }}+p\left(1+(-1)^{k} \cos \delta\right)|k \oplus 1\rangle\langle k \oplus 1|\right]$ with $N_{k}$ being the renormalization constant defined as $N_{k}^{-1}=1+(-1)^{k} p \cos \delta$ and $\oplus$ stands for addition modulo 2 . Then the state-dependent fidelity becomes

$$
F_{m_{k}}(\delta)=1-\frac{p\left(1+(-1)^{k} \cos \delta\right)^{2}}{2\left(1+(-1)^{k} p \cos \delta\right)},
$$

where $k=0,1,2,3$. When $p \rightarrow 1$, the limiting values are calculated as $F_{m_{1,3}}(\delta)=\cos ^{2}(\delta / 2)$ and $F_{m_{0,2}}(\delta)=$ $\sin ^{2}(\delta / 2)$.

For $p \leq 1 / 11$ and $p<1 / 5$, all states can be teleported, respectively, with $F>5 / 6$ and $F>2 / 3$, independent of Alice's measurement result. For the equatorial qubits $\delta=\pi / 2$, we find that as far as $p<1-\sqrt{2} / 2$, teleportation fidelity will surpass that of the PCCM regardless of the measurement outcome. On the other hand, if the qubits are chosen at the neighborhood of $|1\rangle$, then for even $k$ the channel damping rate should be bounded as $0 \leq p \leq$ 0.162 .

Although state-dependent teleportation fidelity is mainly determined by Alice's measurement result, the average fidelity calculated using Eq. (2) is the same for all measurement results and given as

$$
F=\frac{1}{4 p^{2}}\left(2 p+q^{2} \ln \frac{q}{1+p}\right)
$$

which takes the minimum and maximum values of $1 / 2$ and 1 for $p=1$ and $p=0$, respectively.

For the second scenario, the output density operator elements $\rho_{\text {out }}^{(j l)}\left(m_{k}\right)$ are found in terms of the input density operator elements $\rho_{\text {in }}^{(j l)}$ as follows: $\rho_{\text {out }}^{(00)}\left(m_{k}\right)=$ $q \rho_{\text {in }}^{(00)}+\left(1-(-1)^{k}\right) p / 2, \rho_{\text {out }}^{(01)}\left(m_{k}\right)=\sqrt{q} \rho_{\text {in }}^{(01)}, \rho_{\text {out }}^{(10)}\left(m_{k}\right)=$ $\sqrt{q} \rho_{\text {in }}^{(10)}$ and $\rho_{\text {out }}^{(11)}\left(m_{k}\right)=q \rho_{\text {in }}^{(11)}+\left(1+(-1)^{k}\right) p / 2$. When Alice measures $m_{k}$ and applies the appropriate unitary transformation to get the highest fidelity for the process, i.e., when Bob receives the information that $k=3$ or $k=1$ for $\left|\psi^{+}\right\rangle$and $\left|\psi^{-}\right\rangle$respectively, he can use a Z-gate to rotate the state on his side to obtain the above state. Then state-dependent fidelity can be found as

$$
F_{m_{k}}(\delta)=1-\frac{1}{2}\left[p\left(1+(-1)^{k} \cos \delta\right)-x\right],
$$

where $x=(\sqrt{q}-q) \sin ^{2} \delta$. In the limit of $p \rightarrow 1$, we get the same functions as those obtained from Eq. (34).

It is clearly seen from above results that the range of qubits that can be teleported correctly depends not only on the strength of the ADC but also on the measurement result of Alice.
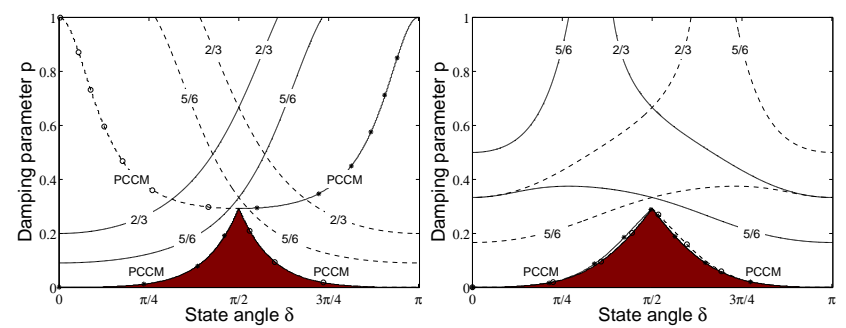

FIG. 3: Optimal state-dependent fidelity in the presence of the ADC when the initial MES is $\left|\psi^{ \pm}\right\rangle$(left) and $\left|\phi^{ \pm}\right\rangle$(right) and both qubits are affected by damping. Contours correspond to $F=2 / 3, F=5 / 6$ and the optimal PCCM fidelities when Alice's measurement result is $|01\rangle\langle 01|$ or $|11\rangle\langle 11|$, solid curves, and when Alice's measurement result is $|10\rangle\langle 10|$ or $|00\rangle\langle 00|$, dotted curves.

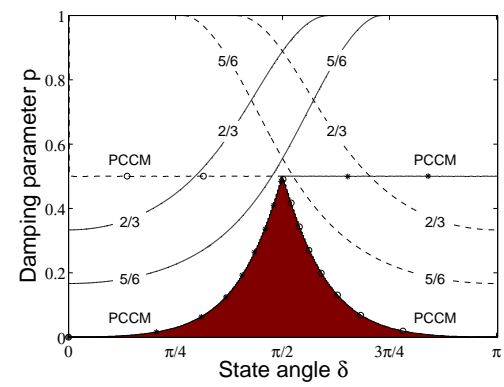

FIG. 4: Same as in Fig. 3 (left) for $\left|\psi^{ \pm}\right\rangle$but for the case when only one of the qubits is affected by the ADC. For $\left|\phi^{ \pm}\right\rangle$, the meaning of curves is reversed.

Results imply that some of the states can be teleported with much better fidelity than others depending on $m_{k}$. This enables Alice and Bob, in a communication protocol, to decide to choose their qubits randomly from a range of states with higher fidelity when a certain measurement result, say $\left\{m_{0}, m_{2}\right\}$, is obtained. As seen in Figs. [3and 4, some states give better fidelity than others depending on $m_{k}$. In the figure we have shaded regions where all the states can be teleported with $F>5 / 6$ regardless of Alice's outcome. Note that the states with $\delta=\pi / 2 \mp \Delta$ can tolerate much higher damping rates than the ones located around the poles of the Bloch sphere. It is also seen that in Scenario 1 it is advantageous to rotate the initial entangled state into $|\phi\rangle$ because it is more immune to the ADC and therefore provides a larger parameter space for $F>5 / 6$ teleportation.

Let us assume that qubits chosen from a range defined by $\Lambda$ have higher fidelity when Alice measures $\left\{m_{0}, m_{2}\right\}$, on the other hand qubits chosen from $\lambda$ have higher fidelity when Alice measures $\left\{m_{1}, m_{3}\right\}$. Then in a teleportation protocol, Alice first mixes her state chosen from $\Lambda$ with her part of the entangled state and makes a measurement, when she obtains $\left\{m_{0}, m_{2}\right\}$, she sends the other qubit of entangled state to Bob together with the classical information, then Bob applies unitary transformation 
to get the desired state. When she gets $\left\{m_{1}, m_{3}\right\}$, either she sends nothing or a dummy state. In this way, they can increase the fidelity of the process. If they decide to abort the protocol whenever Alice measures $\left\{m_{1}, m_{3}\right\}$ then the efficiency of the process is low.

If Alice and Bob decide to keep all measurement results then the fidelity of the process can be written as

$$
F(\delta)=\sum_{k=0}^{3} p_{m_{k}} F_{m_{k}}=q-\frac{x}{2}
$$

where $x$ is defined as in Eq. (36), $F_{m_{k}}$ is the fidelity of the output state to the teleported state when Alice obtains $m_{k}$, and $p_{m_{k}}$ is the probability of obtaining this result. Moreover, if they do that for any $(\delta, \gamma)$, they end up with $F=2 / 3+(2 \sqrt{q}-p) / 6$. From Eq. (36), it can be seen that for a fixed $p$ of the channel, if the state to be teleported is chosen such that $\delta<\pi / 2$, then the set $\left\{m_{1}, m_{3}\right\}$ gives higher teleportation fidelity for that state; otherwise, the set $\left\{m_{0}, m_{2}\right\}$ yields higher fidelity. Let us assume that Alice randomly chooses a state to be teleported from the upper hemisphere of the Bloch sphere, $(\delta<\pi / 2)$, therefore, their preferred measurement set is $\left\{m_{1}, m_{3}\right\}$, which occurs with a probability of $1 / 2$. When the measurement result is $\left\{m_{0}, m_{2}\right\}$, she sends nothing according to the protocol described above. In this way, the fidelity of the process increases to $F(\delta)=F_{m_{1}}(\delta)=F_{m_{3}}(\delta)$ and the average fidelity becomes $F=2 / 3+(4 \sqrt{q}+p) / 12$.

In the same way, if entangled state is distributed by a third party and both qubits undergo damping, Alice proceeds as explained above. If Alice and Bob decide to keep all measurement results then the fidelity of the process becomes

$$
F(\delta)=\sum_{k=0}^{3} p_{m_{k}} F_{m_{k}}=\frac{2-p\left(1+\cos ^{2} \delta\right)}{2\left(1-p^{2} \cos ^{2} \delta\right)},
$$

where $F_{m_{k}}$ is the fidelity of the output state to the teleported state when Alice obtains $m_{k}$, and $p_{m_{k}}$ is the probability of obtaining this result. Moreover, if they do that for any $(\delta, \gamma)$, they end up with $F=\frac{1}{4 p^{2}}\left[2 p+q^{2} \ln \left(\frac{q}{1+p}\right)\right]$. From Eq. (34), it can be seen that for a fixed $p$ of the channel, if the state to be teleported is chosen such that $\delta>\pi / 2$, then the set $\left\{m_{0}, m_{2}\right\}$ gives higher fidelity; otherwise, the set $\left\{m_{1}, m_{3}\right\}$ does. Let us assume that Alice randomly chooses the state to be teleported from the lower hemisphere of the Bloch sphere, $(\delta>\pi / 2)$, therefore, their preferred measurement set is $\left\{m_{0}, m_{2}\right\}$.

\section{Input states $\left|\phi^{ \pm}\right\rangle$}

The output density operators for Alice's outcomes $m_{k=0,1,2,3}$ can be written as

$$
\begin{aligned}
\widehat{\rho}_{\text {out }}\left(m_{k}\right)= & N\left[q \widehat{\rho}_{\text {in }}+p\left(1+(-1)^{k} p \cos \delta\right) \hat{\sigma}_{x}^{k}|0\rangle\langle 0| \hat{\sigma}_{x}^{k}\right. \\
& \left.+(-1)^{k} p q \cos \delta \hat{\sigma}_{x}^{k}|1\rangle\langle 1| \hat{\sigma}_{x}^{k}\right]
\end{aligned}
$$

with $N^{-1}=1+(-1)^{k} p \cos \delta$ from which the state dependent fidelity is derived as

$$
F_{m_{k}}(\delta)=1-\frac{p[3-2 p-(2 p-1) \cos (2 \delta)]}{4\left(1+(-1)^{k} p \cos \delta\right)}
$$

with the limiting values of $F_{m_{0,2}}(\delta)=\cos ^{2}(\delta / 2)$ and $F_{m_{1,3}}(\delta)=\sin ^{2}(\delta / 2)$ for $p$ approaching 1 . It is easy to see that as $p$ approaches $0, F_{m_{k}}(\delta) \rightarrow 1$. For $p<1 / 6$, all states can be teleported with $F>5 / 6$ regardless of the outcome. Average values of teleportation fidelity for these two cases are the same as given in Eq. (35).

For the second scenario, contrary to the first scenario, the output state that Bob gets after the proper application of the quantum gates, and its fidelity to the desired state is the same as that of the case when initial MES is $|\psi\rangle$. When only one of the qubits of the MES goes through the ADC, distributing either $|\psi\rangle$ or $|\phi\rangle$ does not give any advantage to the parties.

\section{B. Phase damping channel}

When the channel is the PDC, given by (23), only the off-diagonal elements are affected by the damping. The fidelity of the teleportation process when the initial MES is subjected to the PDC is independent of Alice's measurement result, because, contrary to the ADC case, Bob can use $\mathrm{X}$ and $\mathrm{Z}$ gates to rotate all the possible outcomes to each other and to the state with the highest fidelity to the input one. The elements of the density matrix can be written as $\rho_{\text {out }}^{(00)}=\rho_{\text {in }}^{(00)}, \rho_{\text {out }}^{(01)}=q^{2} \rho_{\text {in }}^{(01)}, \rho_{\text {out }}^{(10)}=q^{2} \rho_{\text {in }}^{(10)}$ and $\rho_{\text {out }}^{(11)}=\rho_{\text {in }}^{(11)}$ for the first scenario. In case of the second scenario, the elements of the density matrix are the same as above with $q^{2}$ replaced by $q$. Then the fidelity of the teleportation process for scenario 1 can be written as

$$
\begin{aligned}
F(\delta) & =1-\frac{1}{2} p(2-p) \sin ^{2} \delta \\
F & =1-\frac{1}{3} p(2-p)
\end{aligned}
$$

and for scenario 2 as

$$
\begin{aligned}
F(\delta) & =1-\frac{1}{2} p \sin ^{2} \delta, \\
F & =1-\frac{1}{3} p .
\end{aligned}
$$

The effect of the PDC on the teleportation fidelity is the same for both initial MES $|\phi\rangle$ and $|\psi\rangle$. The qubit states with $\delta=0$ and $\delta=\pi$ which are located at the poles of the Bloch sphere are always teleported with $F=1$. Because these states correspond to $|0\rangle$ and $|1\rangle$, which do not carry relative phase information and hence are not affected by the PDC. Indeed, these results show that if Alice chooses the states to be teleported around the poles then they can have a better teleportation fidelity (see Fig. 5). On the 
other hand, states with $\delta=\pi / 2$, which correspond to all the states lying on the equator of the Bloch sphere, are the most affected states.

If Eve does not have the information on the region from which the qubits are chosen, then the best she can do is to use the optimal universal quantum cloning machine of Bužek et al. [13]. Then we find that any qubit state satisfying $\sin ^{2} \delta<1 / 3 p(2-p)$ and $\sin ^{2} \delta<1 / 3 p$, respectively for the first and second scenarios, can be teleported in the presence of PDC with higher fidelity than that of the cloning machine of the eavesdropper. It is apparent that if there is no eavesdropper and that parties just want to beat the classical limit, the range of qubits at a fixed $p$ is much larger.

Now, let us assume that the states to be teleported are chosen with fixed $\delta$ but varying $\gamma$, and the information on $\delta$ may be leaked to an eavesdropper. Since the eavesdropper may use the optimal PCCM, to speak about a secure teleportation its fidelity should exceed the PCCM fidelity given in Eq. (33). Comparing Eq. (33) with state dependent teleportation fidelities for PDC given in Eqs. 41 and 42, we find $\cos \delta<-1+1 / x^{\prime}$ where $x^{\prime}=\sqrt{2}-1+2 p(2-p)$ and $\cos \delta>1-1 / x^{\prime \prime}$ where $x^{\prime \prime}=\sqrt{2}-1+2 p$, respectively for scenarios 1 and 2 . If $\delta$ is chosen in the neighborhood of $|1\rangle$, the damping rate of the channel should satisfy $0 \leq p<(3-2 \sqrt{2}) / 4$ and $0 \leq p<1-(\sqrt{1+2 \sqrt{2}}) / 2$, respectively for the first and second scenarios. On the other hand, if the states to be teleported are chosen from the equatorial qubit states, the damping rate of the channel should satisfy $p<1-1 / 2^{1 / 4}$ and $p<1-1 / \sqrt{2}$, respectively, for the first and second scenarios. These requirements are obviously stricter than those for the universal CM. We see in Fig. 5 that while for the universal cloning machine the constraint on $p$ relaxes as we approach to the poles of the Bloch sphere, for the PCCM it becomes tighter. This is because as we approach the poles, the fidelity of the clones from the PCCM gets closer to one requiring a $\mathrm{PDC}$ with damping rates approaching to zero.

\section{Depolarizing channel}

When the channel is the DC, the elements of the density matrix can be written as $\rho_{\text {out }}^{(00)}=\chi \rho_{\text {in }}^{(00)}+\mu \chi$, $\rho_{\text {out }}^{(01)}=\xi \rho_{\text {in }}^{(01)}, \rho_{\text {out }}^{(10)}=\xi \rho_{\text {in }}^{(10)}$ and $\rho_{\text {out }}^{(11)}=\chi \rho_{\text {in }}^{(11)}+\mu \chi$ for the first scenario, where we have used $\mu=(1-q) / 2$ and $\chi=(1+q)$ and $\xi=q^{2}$. In case of the second scenario, the elements of the density matrix are the same as above with $\chi=1$ and $\xi=q$. Then the fidelity of the teleportation process for the first and second scenarios can be written, respectively, as

$$
\begin{gathered}
F(\delta)=F=\frac{1}{2}+\frac{1}{2} q^{2}, \\
F(\delta)=F=\frac{1}{2}+\frac{1}{2} q
\end{gathered}
$$

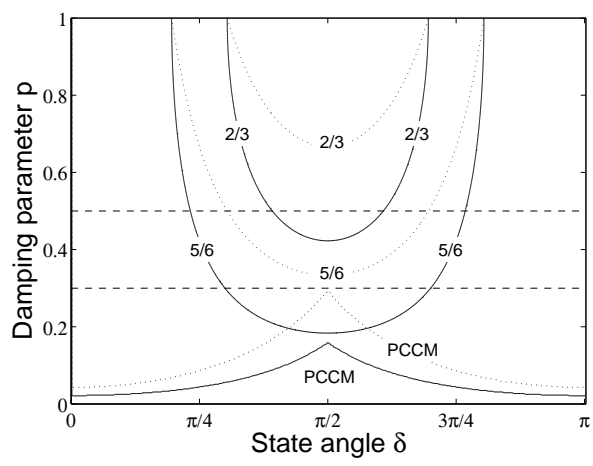

FIG. 5: State-dependent fidelity in the presence of the PDC when the initial MES is any of the Bell states and only one of the qubits (dashed curves) and both qubits (solid curves) are affected by damping. Contours show $F=2 / 3, F=5 / 6$ and the optimal PCCM fidelities. Horizontal dashed lines correspond to the values of damping rate for $f_{\text {ent }}=3 / 4$ for scenarios 1 (lower) and 2 (upper).

from which we see that fidelity is independent of $\left|\psi_{\text {in }}\right\rangle$. For DC too, contrary (similar) to the ADC (PDC), Bob can use quantum gates to rotate all possible outcomes to each other. Therefore, the fidelity is independent of the input state, of Alice's measurement result, and of the initially distributed MES The parties in the protocol can choose their qubits from the whole Bloch sphere and an eavesdropper may use a universal quantum cloning machine, in that case the damping rates of the channels should satisfy $p<1-\sqrt{6} / 3$ and $p<1 / 3$ to surpass the no-cloning limit. In case of an eavesdropper with the PCCM, the relation between the qubits states that can be teleported securely and the damping rate of the channel becomes $\cos \delta<-(1+$ $\sqrt{2})\left(-1+[1+4(\sqrt{2}-1)(x x-1)]^{1 / 2}\right) / 2$ and $\cos \delta<$ $\left(-1+[17-12 \sqrt{2}+8 p(\sqrt{2}-1)]^{1 / 2}\right) /(2 \sqrt{2}-2)$ for the first and second scenarios.

\section{Direct transmission: Noisy state + shared MES}

For Bob to whom Alice wants to teleport the unknown state $\left|\psi_{\text {in }}\right\rangle$, it is difficult to distinguish whether the $\left|\psi_{\text {in }}\right\rangle$ is a noisy state or the quantum channel is responsible for the noise. The state to be teleported might be subjected to noise, loose its coherence and becomes a mixed state before it is teleported.

Let us assume that Alice and Bob share a MES, which they have obtained using entanglement distillation and purification protocols. In this section, we assume that the qubit is influenced by the ADC, PDC and DC, and discuss the outcome of the teleportation process. We assume that only the qubit to be teleported is subjected to noise and the shared entangled state is any of the 
Bell states. Indeed, this is similar to direct transmission scheme where the original state $\left|\psi_{\text {in }}\right\rangle$ is sent directly to Bob through noisy channel.

If $\left|\psi_{\text {in }}\right\rangle$ is subjected only to the ADC, the elements of the output density matrix become $\rho_{\text {out }}^{(00)}=\rho_{\text {in }}^{(00)}+p \rho_{\text {in }}^{(11)}$, $\rho_{\text {out }}^{(01)}=\sqrt{q} \rho_{\text {in }}^{(01)}, \rho_{\text {out }}^{(10)}=\sqrt{q} \rho_{\text {in }}^{(10)}$ and $\rho_{\text {out }}^{(11)}=q \rho_{\text {in }}^{(11)}$ where $\rho_{\text {in }}^{(k l)}$ are the elements of the density matrix of $\left|\psi_{\text {in }}\right\rangle$. Then fidelity is found as

$$
F(\delta)=1-\frac{1}{2}\left[2 p \sin ^{2}(\delta / 2)-(\sqrt{q}-q) \sin ^{2} \delta\right] .
$$

Averaging this over all possible input states, average fidelity is found as

$$
F=\frac{2}{3}+\frac{1}{6}(2 \sqrt{q}-p)
$$

which is the same as for scenario 2, when the entangled state is distributed through the ADC. We see that depending on the damping parameter, the range of qubits that can be teleported with a desired fidelity changes (see Fig. 6). For example when $p=0.8$, when only $\left|\psi_{\text {in }}\right\rangle$ is subjected to noise, the states with $\delta<0.5436 \pi$ and $\delta<0.4021 \pi$ can be teleported, respectively, with $F>2 / 3$ and $F>5 / 6$. For the $\left|\psi_{\text {in }}\right\rangle$ damped case, all the states satisfying $\delta<0.2677 \pi$ can be teleported with $F>5 / 6$. In Fig. 6, we have depicted the fidelity of the PCCM from which we see that when $p=1 / 2$, the teleportation fidelity and the PCCM fidelity are equal for the qubits $0 \leq \delta \leq \pi / 2$. In this range of qubits, a secure teleportation is possible for damping rates $p<1 / 2$. As $\delta$ approaches to $\pi$, the damping rate $p$ approaches to zero to achieve secure teleportation.

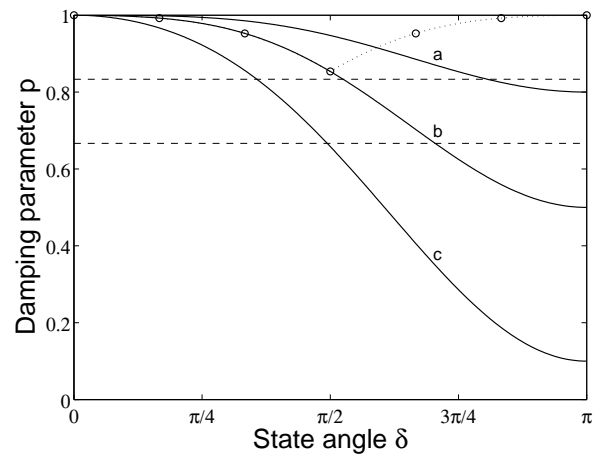

FIG. 6: State dependent teleportation fidelity when the qubit to be teleported is subjected to the ADC, a, $p=0.2$, b, $p=0.5$, and c, $p=0.9$. Horizontal dotted lines denote the limits between classical and quantum operations (lower), and the secure quantum teleportation (upper). The curve marked with circles corresponds to the optimal PCCM fidelity.

When the qubit is subjected only to the PDC, the output density operator becomes $\hat{\rho}_{\text {out }}=q \hat{\rho}_{\text {in }}+$ $p\left[\cos ^{2}(\delta / 2)|0\rangle\left\langle 0\left|+\sin ^{2}(\delta / 2)\right| 1\right\rangle\langle 1|\right]$, resulting in a fidelity $F=1-p \sin ^{2}(\delta / 2)$ from which average fidelity can be
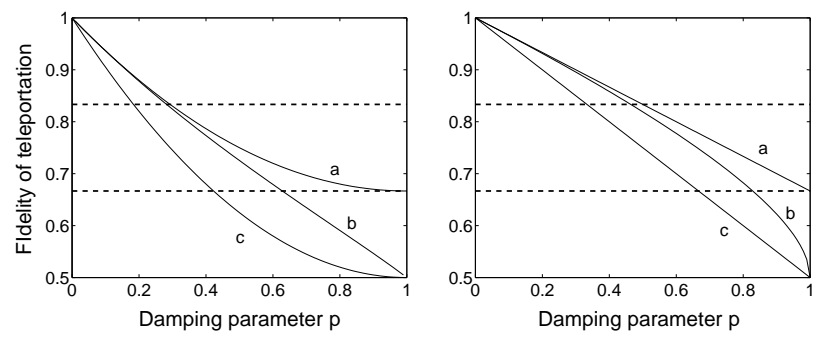

FIG. 7: Average fidelity with noisy channels: a, PDC, b, $\mathrm{ADC}$, and c, DC for scenarios 1 (left) and 2 (right). Horizontal dashed lines denote the limits between classical and quantum operations (lower), and the secure quantum teleportation (upper).

written as $F=1-p / 3$. Comparing these equations with Eq. (42), it is seen that when the PDC affects only the qubit to be teleported, the fidelity is the same as in scenario 2 when the distributed entangled states undergo the PDC. We observe the same similarity if only the qubit $\left|\psi_{\text {in }}\right\rangle$ is subjected to DC. In this case the fidelity expression is given as in Eq. (44).

In the analysis of security of a damping particular channel, the fidelities of optimal cloning machines were taken as a reference: either (i) the optimal universal cloning machine if no a priori information about a teleported state is given or (ii) the optimal phase-covariant cloning machine if prior partial information about the state is available. Clearly, a channel is secure if it provides a better fidelity than the optimal cloning. This is the lowest fidelity bound for security of any channel assuming that Alice sends her qubit through a damping channel, while an eavesdropper copies qubit at Alice's site and does not send it (or sends it through a perfect channel). Otherwise, the action of the channel will restrict the quality of the cloning consistent with the channel and, thus, less demanding security conditions can be given.

\section{CONCLUSION}

We have examined the problem of teleportation fidelity in the presence of various types of noise during the entanglement distribution of the teleportation process. Using the fully entangled fraction and concurrence, we derived the bounds on the damping parameters of channels so that the average fidelity (i) exceeds the classical limit, and (ii) satisfy the security condition for teleportation. Moreover, we derived the range of states that can be teleported accurately with a desired fidelity value and studied how this range is affected by noise. For the security condition, we considered eavesdroppers with universal and phase-covariant cloning machines where the first eavesdropper has no information on the qubit to be teleported but in the latter he/she knows the $\delta$ but not the relative phase $\gamma$. 
For the ADC, although the bounds on $p$ for one-qubit affected case are the same for both $\left|\psi^{ \pm}\right\rangle$and $\left|\phi^{ \pm}\right\rangle$as the source entangled state, for the two-qubit affected case we find that the bounds are different and much tighter for $\left|\psi^{ \pm}\right\rangle$. This implies that if one is given $\left|\psi^{ \pm}\right\rangle$, instead of distributing this state directly, it is better to first locally convert to $\left|\phi^{ \pm}\right\rangle$and distribute it. In that case the effect of damping is less pronounced. We observe that only for the ADC these bounds change with the initial MES to be distributed. We have found that contrary to the case of the $\mathrm{ADC}$, in the presence of the PDC and $\mathrm{DC}$, two-qubit affected case cannot be made to have higher entangled fraction than the one-qubit affected case. Hence, the average fidelity cannot be increased by subjecting one of the qubits to controlled dissipation. As seen in Fig. 7 average fidelity is dependent on the type and strength of damping in the channel. For the PDC, fidelity is always larger than $2 / 3$ if $p \neq 1$, on the other hand for the ADC and DC average fidelity decreases below $2 / 3$ down to $1 / 2$ depending on the damping rate.

We have discussed the direct transmission case, too. We observe that the results obtained for direct transmission and teleportation with one-qubit affected entanglement distribution case (scenario two), are the same in the cases of the DC and PDC. However, discrepancies are seen for the case of the ADC. Average fidelity for scenario 2 is more immune to damping than the direct transmission.

This study shows that information on the noise affecting the teleportation process during the phases of entanglement distribution and the qubit preparation can be helpful in increasing the fidelity. Moreover, it is important to note that if the source of noise in the process is not known then all should be attributed to an eavesdropper. Thus, the criterion on the teleportation fidelity should be re-formulated taking into account the set of states from where to be teleported state is chosen and the optimal cloning machine for that set.

\section{Acknowledgments}

We thank Prof. Nobuyuki Imoto and Prof. Masato Koashi for their useful comments. AM was supported by the Polish Ministry of Science and Higher Education under Grant No. 1 P03B 06428.
[1] R. Horodecki, P. Horodecki, M. Horodecki and K. Horodecki, to appear in Rev. Mod. Phys. (2007), e-print quant-ph/0702225

[2] V. Scarani et al., Rev. Mod. Phys. 77, 1225 (2005); N.J. Cerf and J. Fiurášek, in Progress in Optics 49, edited by E. Wolf (Elsevier, Amsterdam, 2006), pp. 455-545.

[3] C.H. Bennett et al., Phys. Rev. Lett 70, 1895 (1993).

[4] H.F. Hofmann, Phys. Rev. A 66, 032317 (2002).

[5] S. Popescu, Phys. Rev. Lett. 72, 797 (1994); N. Gisin, Phys. Lett. A 210, 157 (1996); R. Horodecki and M. Horodecki, Phys. Rev. A 54, 1838 (1996).

[6] M. Horodecki, P. Horodecki and R. Horodecki, Phys. Rev. A 60, 1888 (1999); R. Horodecki, M. Horodecki and P. Horodecki, Phys. Lett. A 222, 21 (1996)

[7] F. Grosshans and P. Grangier, Phys. Rev. A 64, 010301(R) (2001).

[8] S. Bandyopadhyay, Phys. Rev. A 65, 022302 (2002).

[9] G.G. Carlo, G. Benenti, G. Casati and C. MejiaMonasterio, Phys. Rev. A 69, 062317 (2004).

[10] G. Gordon and G. Rigolin, Phys. Rev. A 73, 042309 (2006).

[11] W.K. Wootters and W.H. Zurek, Nature 299, 802 (1982); D. Dieks, Phys. Lett. A 92, 271 (1982).
[12] M.A. Nielsen and I.L. Chuang, Quantum Computation and Quantum Information (Cambridge University Press, 2000).

[13] V. Bužek and M. Hillery, Phys. Rev. A 54, 1844 (1996).

[14] N. Gisin and S. Massar, Phys. Rev. Lett. 79, 2153 (1997).

[15] V. Bužek and M. Hillery, Phys. Lett. 81, 5003 (1998).

[16] D. Bruß et al., Phys. Rev. A 57, 2368 (1998).

[17] C.H. Bennett et al., Phys. Rev. Lett. 76, 722 (1996).

[18] P. Badzia̧g, M. Horodecki, P. Horodecki and R. Horodecki, Phys. Rev. A 62, 012311 (2000).

[19] C.H. Bennett, D.P. DiVincenzo, J.A. Smolin, W.K. Wootters, Phys. Rev. A 54, 3824 (1996).

[20] J. Preskill, Lecture Notes for Physics 219: Quantum Information and Computation (Caltech, Pasadena, California, 1999), www. theory. caltech. edu/ people/ preskill/ ph229.

[21] W. K. Wootters, Phys. Rev. Lett. 80, 2245 (1998).

[22] D. Bruß, M. Cinchetti, G.M. D'Ariano and C. Macchiavello, Phys. Rev. A 62, 012302 (2000).

[23] J. Fiurášek, Phys. Rev. A 67, 052314 (2003).

[24] Jiangfeng Du et al., Phys. Rev. Lett. 94, 040505 (2005). 